\title{
miR-125b, miR-200c Are Correlated with the Severity of Interstitial Lung Disease in Dermatomyositis/Polymyositis
}

\author{
Zhen Jiang1, Jinhui Tao², Xiangpei Li ${ }^{2 *}$ \\ ${ }^{1}$ Department of Rheumatology and Immunology, Huai'an First People's Hospital, Nanjing Medical University, Huai'an, China \\ ${ }^{2}$ Department of Rheumatology and Immunology, Affiliated Anhui Provincial Hospital, Anhui Medical University, Hefei, China \\ Email: *lixiangpei55@126.com
}

How to cite this paper: Jiang, Z., Tao, J.H. and Li, X.P. (2018) miR-125b, miR-200c Are Correlated with the Severity of Interstitial Lung Disease in Dermatomyositis/Polymyositis. Open Journal of Rheumatology and Autoimmune Diseases, 8, 1-16. https://doi.org/10.4236/ojra.2018.81001

Received: December 28, 2017

Accepted: January 28, 2018

Published: February 1, 2018

Copyright $\odot 2018$ by authors and Scientific Research Publishing Inc. This work is licensed under the Creative Commons Attribution International License (CC BY 4.0).

http://creativecommons.org/licenses/by/4.0/ (c) (i) Open Access

\begin{abstract}
Objective: To explore the correlations between miR-125b, miR-200c, and the severity of interstitial lung disease associated with dermatomyositis/polymyositis (DM/PM-ILD). Methods: 30 consecutive patients with DM/PM and 23 healthy controls were recruited into current study. Anti-JO-1, anti-SSA, muscle enzymes, the data of chest HRCT and pulmonary function test were collected. 9 consecutive DM/PM-ILD patients underwent bronchoalveolar lavage (BAL). TGF- $\beta 1$ and surfactant protein D (SP-D) in BAL fluid (BALF) and plasma were detected by ELISA. miR-125b and miR-200c in PBMCs and bronchoalveolar cells were detected by QRT-PCR. All patients were classified into three groups: Mild or non-ILD group, moderate ILD group, and severe ILD group. The correlations between miRNAs and the severity of ILD, the lung damage markers, auto-antibodies, were analyzed. Results: The levels of miR-125b and miR-200c in bronchoalveolar cells were higher than in PBMCs, and the levels of TGF- $\beta 1$ and SP-D were higher in BALF than in plasma in $\mathrm{DM} / \mathrm{PM}$-ILD patients. There were positive correlations between miR-125b, miR-200c in bronchoalveolar cells and SP-D in BALF. The levels of miR-125b and miR-200c in severe ILD group were higher than in mild or non-ILD and moderate ILD groups. There were negative correlations between miR-125b, miR-200c, and FEV1, and between miR-200c and DLCO. The patients with anti-JO-1 antibody had higher levels of miR-125b and miR-200c, and had more severe condition of ILD. Conclusion: miR-125b and miR-200c were positively correlated with the lung damage and severity of ILD in DM/PM, which could be important markers for judgement of disease condition in clinic.
\end{abstract}




\section{Keywords}

Dermatomyositis/Polymyositis, Interstitial Lung Disease, miR-125b, miR-200c, Severity

\section{Introduction}

Interstitial lung disease (ILD) is often shown as fibrosis and deposit of extracellular matrix in high resolution computerized tomography (HRCT) and pathology. Epithelial-mesenchymal transition (EMT), involving transition of alveolar epithelial cells (AECs) to pulmonary fibroblasts (or myofibroblasts), and the excessive proliferation of pulmonary fibroblasts, are considered key processes that result in pulmonary fibrosis [1] [2].

MicroRNAs (miRNAs) are small non-coding RNAs that are generally believed involved in regulating the expression of protein-encoding genes by blocking the translation or inducing degradation of target mRNAs [3]. Up to now, miRNAs have been confirmed to play important roles in diverse fibrotic diseases (including lung fibrosis) [4] [5] [6] [7]. miR-200c plays a crucial role in keeping the phenotype of AECs and inhibiting of lung fibrosis [8]. MiR-200c is significantly down-regulated in the lungs of mice with experimental lung fibrosis and its expression was also reduced in the lungs of patients with idiopathic pulmonary fibrosis (IPF) [8]. Moreover, introduction of miR-200c alleviates experimental lung fibrosis by inhibiting of EMT in mice [8]. MiR-125b has been shown disease-suppressing properties and disease-promoting functions in different diseases by targeting different protein-coding genes [9]. Moreover, miR-125b protected the lung in model mouse of acute lung injury by reducing the counts of total cells, proinflammatory cytokines and chemokines in bronchoalveolar lavage fluid (BALF) [10]. In these diseases, miR-125b works mainly through affecting proliferation, differentiation, metastasis of different types of cells.

ILD associated with dermatomyositis/polymyositis (DM/PM-ILD) is one of the most important factors that cause the poor prognosis. ILD in DM/PM proceeds rapidly and the condition is usually severe, such as rapidly progressive ILD (RP-ILD) [11] [12]. We didn't find the reports about the correlation between miR-200c, miR-125b and ILD in DM/PM.

In current study, we reported the association between miR-125b, miR-200c and clinical parameters associated with ILD in DM/PM, such as chest HRCT, pulmonary function test (PFT), and the parameters of lung damage, surfactant protein $\mathrm{D}$ (SP-D) and transforming growth factor beta1 (TGF- $\beta 1$ ).

\section{Materials and Methods}

\subsection{Study Population}

The consecutive inpatients and outpatients were recruited into current study from a tertiary teaching hospital, Anhui Provincial Hospital, Hefei, China, from 
January to September of 2013. All patients satisfied Bohan and Peter criteria for $\mathrm{DM}$ and PM, and had no infection and (or) tumor. According to the definition of ILD on HRCT from the Nomenclature Commitee of the Fleischner Society [13], the patients were judged with ILD or without ILD. 23 healthy persons whose ages and sexes were matched with patients were also included. The study was approved by ethics committee of the hospital which conforms to the provisions of Declaration of Helsinki in 1995 (as revised in Edinburgh 2000). All subjects agreed to participate in current study and signed the agreement.

All patients were arranged for testing muscle enzymes (creatine phosphokinase (CPK), lactate dehydrogenase (LDH)), anti-Jo-1(immunoblotting), and anti-SSA (immunoblotting). We didn't test the other anti-synthetase antibodies because of lower positive rates and the small sample. According to disease activity (based on clinical symptoms, CPK, LDH, bilateral thigh muscle MRI) and severity (based on pulmonary function test and chest HRCT) and condition (acute or chronic) of ILD, those patients were arranged for different therapeutic schedules.

All patients were arranged for chest HRCT and pulmonary function test (PFT). The devices were described in our published article [14]. An independent radiologist read the films. The parameters (FEV1forced expiratory volume in 1 second, DLCO) of pulmonary function were described as a percentage of the measured value/the predicted value.

\subsection{MicroRNA Detection}

All patients were arranged for detection of miR-200c and miR-125b in peripheral blood mononuclear cells (PBMCs) by real-time quantitative reverse transcription-polymerase chain reaction (QRT-PCR). Whole blood samples $(10 \mathrm{~mL})$ taken from cubital vein were injected into tubes with Ethylene Diamine Tetraacetic Acid (EDTA). Anticoagulant blood was centrifugated for five minutes to obtain plasma for detection of TGF- $\beta 1$ and SP-D. PBMCs were isolated from whole blood samples by density gradient centrifugation with Lymphocyte Separation Medium (Tian Jin Hao Yang Biological Manufacture), and kept in TRIzol Reagent (Invitrogen). These PBMCs with TRIzol Reagent were lysed in $-80^{\circ} \mathrm{C}$. The detailed methods of detecting of miR-125b and miR-200c were described in our published article [14].

\subsection{Bronchoalveolar Lavage}

9 patients with ILD who agreed to participate and signed the agreement were arranged for BAL. $80 \mathrm{~mL}$ sterile $0.9 \%$ saline was introduced to the right middle lobe in twice via an electronic bronchoscope. The negative pressure of extraction was $80 \mathrm{mmHg}$. The BALF was filtered through two layers of gauze to remove mucus and centrifuged at $400 \times \mathrm{g}$ for 10 minutes to collect cells to detect miRNAs by QRT-PCR. Bronchoalveolar cells (lysed in TRIzol Reagent) and the supernatant were preserved in $-80^{\circ} \mathrm{C}$. 


\subsection{Detection of TGF- $\beta 1$ and SP-D}

TGF- $\beta 1$ (human TGF- $\beta 1$ ELISA kit, Jiamay Biotech), and SP-D (human Surfactant Protein D ELISA kit, BioVendor) in plasma and the supernatant of BALF were detected by ELISA according to the description of the manufacturers.

\subsection{The Correlation between miR-125b, miR-200c, and the Severity of ILD}

According to the results of chest HRCT and PFT, the patients were classified into three groups: mild or non-ILD group, moderate ILD group and severe ILD group. The patients were regarded as having mild ILD if they had ground glass opacities (GGO), irregular linear opacities, centrilobular nodule, or patched consolidation opacities in HRCT, and the parameters of more than $60 \%$ but less than $80 \%$ for FEV1 and DLCO in PFT. The patients were regarded as having moderate ILD if they had scattered and multiple patches, cord-like opacity, or reticular opacity in lower lobes of two lungs, and the parameters of more than $40 \%$ but less than $60 \%$ for FEV1 and DLCO in PFT. The patients were regarded as having severe ILD if they had UIP (usual interstitial pneumonitis) pattern, reticular opacities, or honeycombing in two lungs in HRCT, and the parameters of less than $40 \%$ for FEV1, and DLCO in PFT.

\subsection{Statistical Analysis}

Kruskal-Wallis test was used to analyze the difference of miR-125b and miR-200c among three level ILD groups. Mann-Whiney test was used to analyze the difference of miR-125b and miR-200c in PBMCs between two groups. Wilcoxon Signed Ranks test was performed to compare the difference of miR-125b and miR-200c between BALF and PBMCs. Spearman correlation was performed to analyze the correlation between miR-125b, miR-200c, and FEV1, DLCO, and between miR-125b, miR-200c, and TGF- $\beta 1$ and SP-D in BALF. GraphPad Prim 5 was used for statistical analysis and plotting. $P$ value $<0.05$ was set for the levels of these tests.

\section{Results}

\subsection{General Information}

30 patients were included in the current study.The mean (standard deviation, $\mathrm{SD}$ ) of age was $42.7 \pm 15.0$ years. The median (interquartile range, IQR) of disease course was 15 (9 - 60) months. Those patients included 11 males and 19 females, $27 \mathrm{DM}$ and 3 PM patients, 25 patients with ILD and 5 patients without obvious ILD.

\subsection{The Correlation between Muscle Enzymes and the Severity of ILD}

According to the definition above mentioned, 9 patients were classified into mild 
or non-ILD group (group 1), 10 patients into moderate ILD group (group 2), and 11 patients into severe ILD group (group 3). The difference of CPK was significant in statistic among three groups $(P=0.0321$, Kruskal-Wallis test), and the level in group 1 was significantly higher than in group $2(P=0.007$, Mann Whitney test), and there was no difference between group 2 and group 3 , and between group 1 and group 3 ( $P=0.1234,0.8148$, respectively, Mann Whitney test) (Supplement Figure S1(a)). The difference of LDH was not significant in statistic among three groups $(P=0.7211$, Kruskal-Wallis test) (Supplement Figure $\mathrm{S} 1(\mathrm{~b}))$.

\subsection{The Correlations between miR-125b, miR-200c and Muscle Enzymes}

According to CPK level and muscle MRI, there were 18 CADM patients (clinical amyopathic dermatomyositis, typical rash but CPK less than $300 \mathrm{IU} / \mathrm{L}$ ) group, 9 DM patients (typical DM, typical rash and CPK more than $300 \mathrm{IU} / \mathrm{L}$ ) group, and 3 PM patients (typical PM, no rash and CPK more than $300 \mathrm{IU} / \mathrm{L}$ ) group.

All 30 patients underwent testing of miR-125b and miR-200c in PBMCs. There was no significant correlation between CPK and LDH, and miR-125b ( $P=$ $0.531,0.348$ respectively, Spearman correlation), and no significant correlation between $\mathrm{CPK}$ and $\mathrm{LDH}$, and miR-200c in PBMCs $(P=0.531,0.541$ respectively, Spearman correlation). There was no significant difference about the levels of miR-125b and miR-200c between CADM group and non-CADM group (typical DM plus typical PM) $(P=0.5393,0.8989$ respectively, Mann-Whitney test), and between CADM and typical DM groups $(P=0.1134,0.3309$ respectively, Mann-Whitney test).

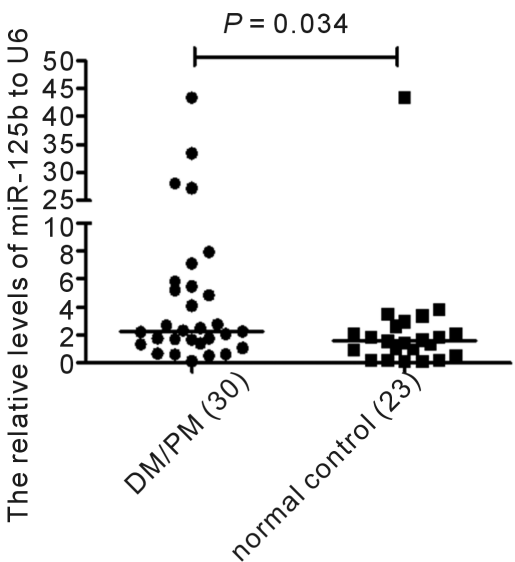

(a)

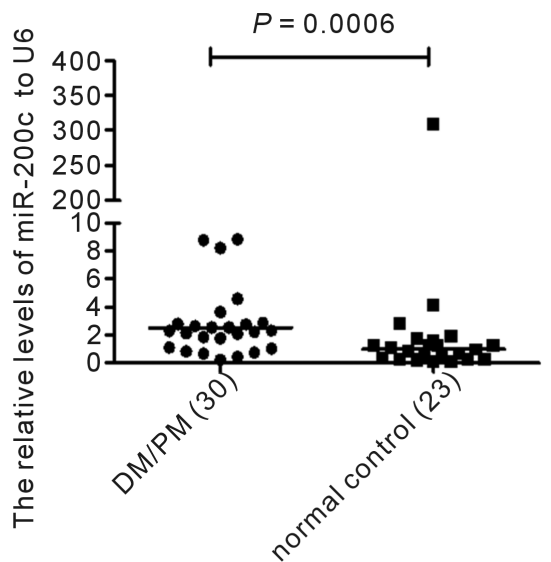

(b)

Figure 1. The relative levels of miR-125b and miR-200c in PBMCs in DM/PM patients and normal control. The levels of miRNAs were detected by QRT-PCR. The relative levels of miR-125b and miR-200c in PBMCs were significantly higher in DM/PM patients than in normal controls $(P=0.034,0.006$, respectively, Mann-Whiney test) (Figure 1 (a), Figure $1(\mathrm{~b}))$. 


\subsection{The Correlations between miR-125b, miR-200c and Treatment}

6 patients were given only glucocorticoid, 1 patient only hydroxychloroquine, 13 patients glucocorticoid plus one kind of disease modifying antirheumatic drug (DMARD), and 10 patients glucocorticoid plus two or more kinds of DMARDs.

There was no significant difference about the levels of miR-125b and miR-200c between only glucocorticoid and only hydroxychloroquine group, and glucocorticoid plus one kind of DMARD group and glucocorticoid plus two or more kinds of DMARDs group ( $P=0.9577,0.6649$ respectively, Mann-Whitney test) (data not shown). According to the amounts of used glucocorticoid and immunosuppressor, 23 patients were classified into "active patients" group (glucocorticoid plus one or two or more kinds of DMARDs), and the other 7 patients into "inactive" one (only glucocorticoid or only hydroxychloroquine). We didn't find the difference of miR-125b and miR-200c between two groups ( $P=$ 1.0000, 0.9391 respectively, Mann-Whitney test) (data not shown).

\subsection{The Relative Levels of miR-125b and miR-200c in PBMCs Were Higher in DM/PM Patients than in Normal Controls}

The relative levels of miR-125b were $1.59(0.57$ - 2.64) and 2.27 (1.36 - 5.56) respectively for normal control and DM/PM groups, and the difference was significant $(P=0.034$, Mann-Whitney test) (Figure 1(a)). The relative levels of miR-200c were $1.00(0.30-1.65)$ and $2.51(1.59-8.34)$ respectively for normal control and DM/PM groups, and the difference was also significant $(P=0.0006$, Mann-Whitney test) (Figure 1(b)).

\subsection{The Relative Levels of miR-200c and miR-125b in Bronchoalveolar Cells Were Higher than Those in PBMCs in DM/PM-ILD Patients}

9 (6 DM-ILD and 3 PM-ILD) of the 25 DM/PM-ILD patients agreed to participate for BAL. The level of miR-125b in bronchoalveolar cells was significantly higher than in PBMCs (1.19 (0.83 - 1.75) vs 0.31 (0.05 - 0.65), $P=0.0188$, Wilcoxon Signed Ranks test) (Figure 2(a)). The level of miR-200c in bronchoalveolar cells was also higher than in PBMCs (6.03 (1.33 - 39.40) vs 0.73 (0.06 - 1.07), $P=0.0078$, Wilcoxon Signed Ranks test) (Figure 2(b)).

\subsection{DM/PM-ILD Patients Had Higher Levels of TGF- $\beta 1$ and SP-D in BALF than in Plasma}

In order to know the correlations between TGF- $\beta 1$, SP-D and ILD in DM/PM, we compared the BALF and plasma levels of the two lung injury markers. The concentration of TGF- $\beta 1$ is 525.79 (492.67 - 651.76) $\mathrm{ng} / \mathrm{mL}$ in BALF, which was significantly higher than in plasma $(35.59(18.02-72.25) \mathrm{ng} / \mathrm{mL})(P=0.0009$, Wilcoxon Signed Ranks test) (Figure 3(a)). The same result was found for SP-D concentration (452.12 (250.08 - 732.36) ng/mL in BALF and 201.14 (48.73 259.32) ng/mL in plasma, $P=0.0177$, Wilcoxon Signed Ranks test) (Figure 3(b)). 


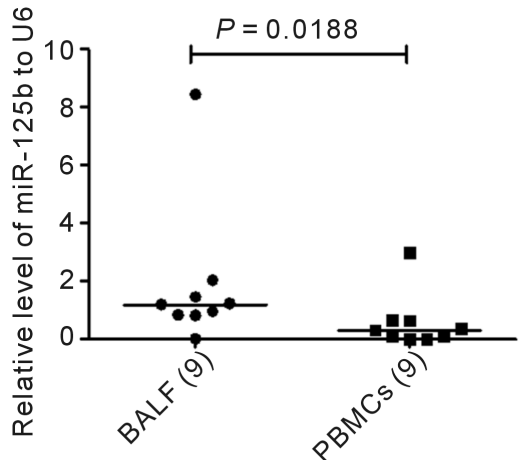

(a)

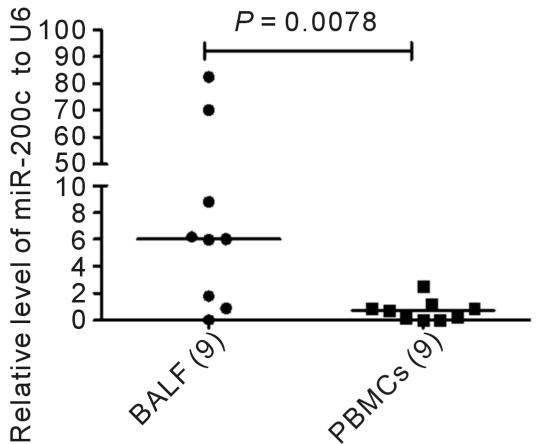

(b)

Figure 2. The relative levels of miR-125b and miR-200c in bronchoalveolar cells and PBMCs in DM/PM-ILD patients. The levels of miRNAs were detected by QRT-PCR. The relative levels of miR-125b and miR-200c in bronchoalveolar cells were higher than those in PBMCs, and the differences were significant $(P=0.0188,0.0078$, Wilcoxon Signed Ranks test) (Figure 2(a), Figure 2(b)).

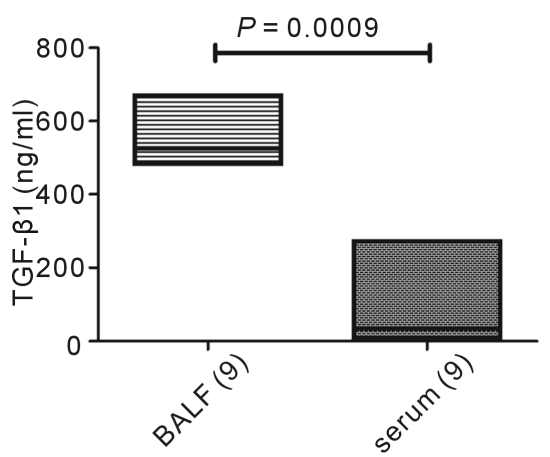

(a)

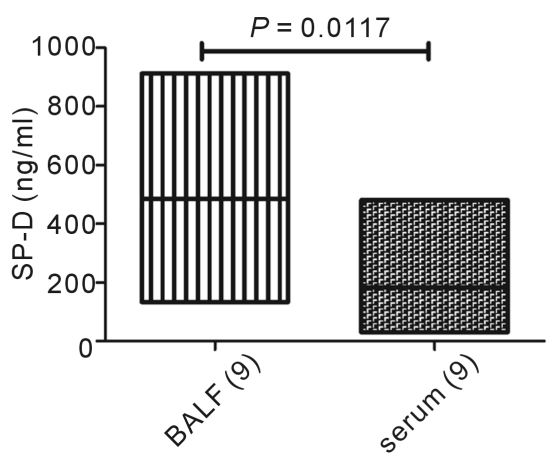

(b)

Figure 3. The levels of TGF- $\beta 1$ and SP-D in BALF and plasma in DM/PM-ILD patients. The concentrations of TGF- $\beta$ land SP-D in BALF and plasma were detected by ELISA.The concentrations of TGF- $\beta 1$ and SP-D in BALF were significantly higher than those in plasma $(P=0.0009,0.0117$, respectively, Wilcoxon Signed Ranks test) (Figure 3(a), Figure 3(b)).

\subsection{The correlations between miR-125b, miR-200c in Bronchoalveolar Cells and TGF- $\beta 1$, SP-D in Supernatant of BALF}

Positive correlations between miR-125b, miR-200c in bronchoalveolar cells and SP-D in BALF were found $(P=0.0083,0.002, \mathrm{r}=0.8333,0.9$ respectively, Spearman correlation) (Figure 4(a), Figure 4(b)). However, there was no significant correlation between miR-125b, miR-200c in bronchoalveolar cells and TGF- $\beta 1$ in BALF (data not shown).

\subsection{The Correlations between miR-125b, miR-200c and Autoantibodies, Anti-SSA, Anti-J0-1}

All 30 patients underwent anti-SSA and anti-JO- 1 tests. The numbers of patients with positive antibodies were $3(10 \%)$ and $3(10 \%)$ respectively for anti-SSA and 


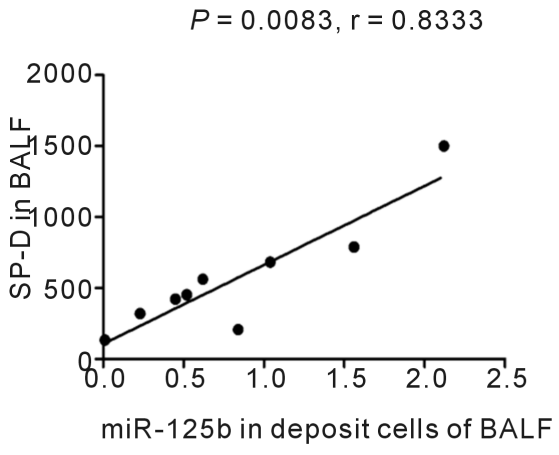

(a)

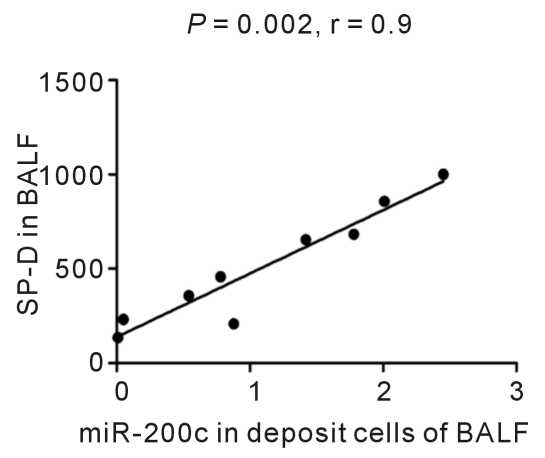

(b)

Figure 4. The correlations between miR-125b, miR200c in bronchoalveolar cells and SP-D in supernatant of BALF in DM/PM-ILD patients. 9 DM/PM-ILD patients were involved for analysis of the correlations between miR-125b, miR200c in bronchoalveolar cells and SP-D in supernatant of BALF. The levels of miR-125b and miR-200c were detected by QRT-PCR, and they were expressed as relative levels to U6. The concentrations of TGF- $\beta$ land SP-D were detected by ELISA. There were positive correlations between miR-125b, miR-200c and SP-D $(P=0.0083,0.002, r=0.8333,0.9$, respectively for miR-125b and miR-200c, Spearman correlation) (Figure 4(a), Figure 4(b)).

anti-JO-1. In patients with anti-JO-1 antibody, the relative levels of miR-125b were $5.47,7.92,27.16$, and the median was 7.92 , which is higher than the whole median $(2.27(1.36-5.56))$ of the 30 patients; the relative levels of miR-200c were $8.83,8.19,22.88$, and the median was 8.83 , which is also higher than the whole median $(2.42(1.59$ - 8.34)). In patients with anti-SSA antibody, the relative levels of miR-125b were $2.06,1.66,33.39$, and the median was 2.06 , which seems not higher than the whole median $(2.27$ (1.36 - 5.56)); the relative levels of miR-200c were $1.83,2.79,44.95$, and the median was 2.79 , which seems not significantly higher than the whole median $(2.42(1.59-8.34))$.

\subsection{The Correlations between Anti-J0-1, Anti-SSA, and the Severity of ILD}

The patients with anti-JO-1 or anti-SSA were all diagnosed with ILD according to chest HRCT, but the patients with anti-JO-1 had more severe condition (more reticular opacity, cord shadow foci and honeycombing foci) than the patients with anti-SSA (more ground-glass opacity, bulla). In patients with anti-JO-1 antibody, FEV1 were 70, 66, 67, and DLCO were 33.52, 21.2, 44.99, respectively. The two parameters were all less than normal values (more than 80 ). However, in patients with anti-SSA antibody, FEV1 were 107, 93, 88, and DLCO were $102.77,85.3,63.9$, respectively. Most of the values were normal.

\subsection{1. miR-125b, miR-200c and the Severity of ILD}

According to the results of HRCT and pulmonary function, 9 patients were classified into mild or non-ILD group (group1), 10 patients into moderate ILD group (group 2), and 11 patients into severe ILD group (group 3). The levels of miR-125b were elevated gradually from group 1 to group 3 , and the difference 
was significant ( $P=0.0088$, Kruskal-Wallis test). The level of miR-125b in group 3 was significantly more higher than in group 1 and group $2(P=$ 0.0107, 0.0133, Mann Whitney test), and the difference was not significant between group1 and group 2 (Figure 5(a)). The levels of miR-200c were also elevated gradually from group 1 to group 3, and the difference was significant ( $P$ $=0.0016$, Kruskal-Wallis test). The level of miR-200c in group 3 was significantly more higher than in group 1 and group $2(P=0.0024,0.0083$, Mann Whitney test), however, the difference was not significant between group1 and group 2 (Figure 5(b)).

\subsection{The Correlations between miR-125b, miR-200c and Pulmonary Function}

There were negative correlations between miR-125b, miR-200c, and FEV1 $(P=$ $0.021,0.041, \mathrm{r}=-0.489,-0.438$, Spearman correlation) (Figure 6(a), Figure $6(\mathrm{~b}))$. There was also negative correlation between miR-200c and DLCO $(P=$ $0.023, \mathrm{r}=-0.483$, Spearman correlation), however, there was no significant correlation between miR-125b and DLCO $(P=0.300, r=-0.231$, Spearman correlation) (Figure 6(c), Figure 6(d)).

\section{Discussion}

DM/PM-ILD often results in more poor prognosis because of no definitely effective therapy to ILD up to now. So, it is very crucial to explore the factors related to ILD. In current study, we found miR-125b and miR-200c, two miRNAs

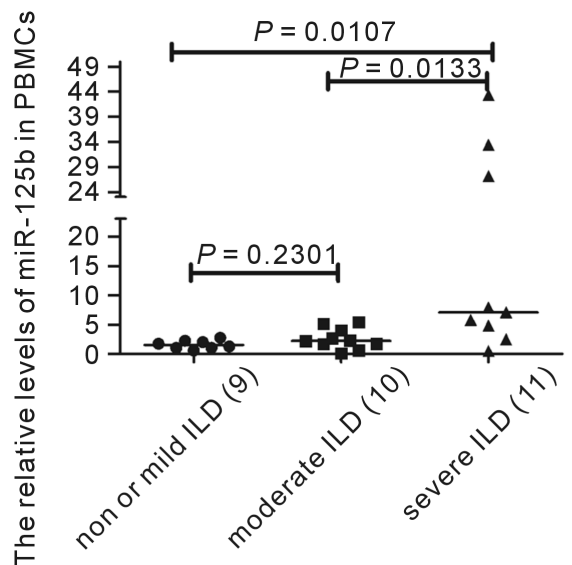

(a)

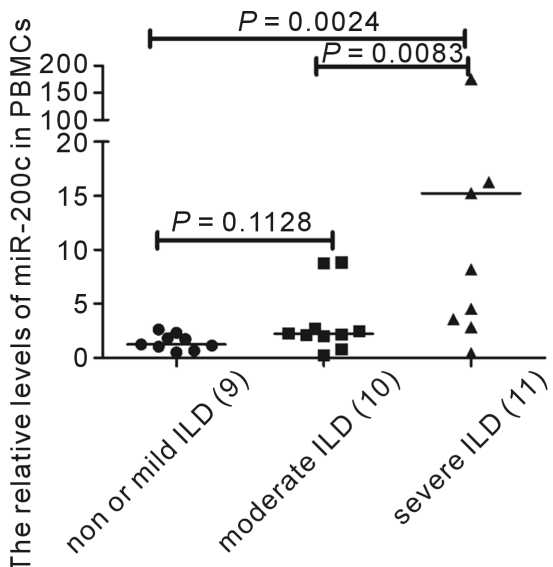

(b)

Figure 5. MiR-125b, miR-200c and the severity of ILD. The levels of miRNAs were detected by QRT-PCR, and they were expressed as relative levels to U6. The difference of miR-125b was significant among three groups of severity for ILD $(P=0.0088$, Kruskal-Wallis test). The level of miR-125b in severe ILD group was significantly more higher than in mild or non-ILD group and moderate ILD group $(P=0.0107,0.0133$, Mann Whitney test) (Figure 5(a)). The difference of miR-200c was significant $(P=$ 0.0016 , Kruskal-Wallis test). The level of miR-200c in severe ILD group was significantly more higher than in mild or non-ILD group and moderate group $(P=0.0024,0.0083$, Mann Whitney test) (Figure 5(b)). 


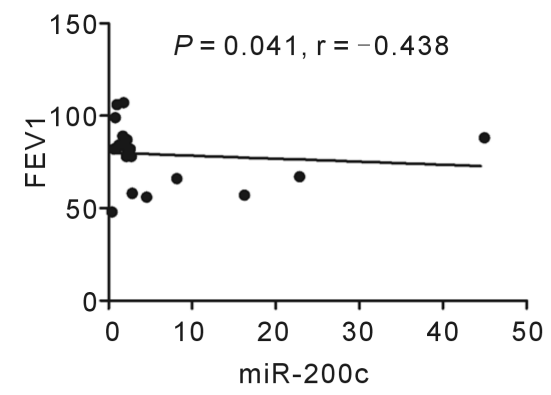

(a)

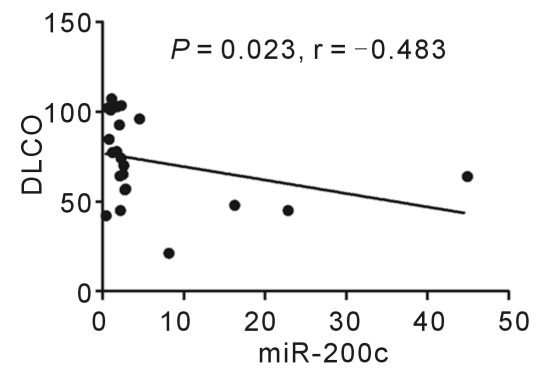

(c)

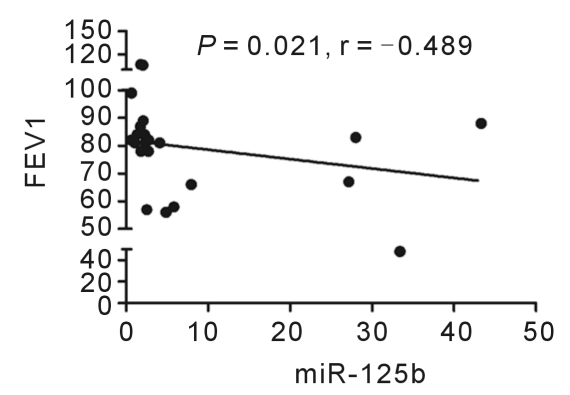

(b)

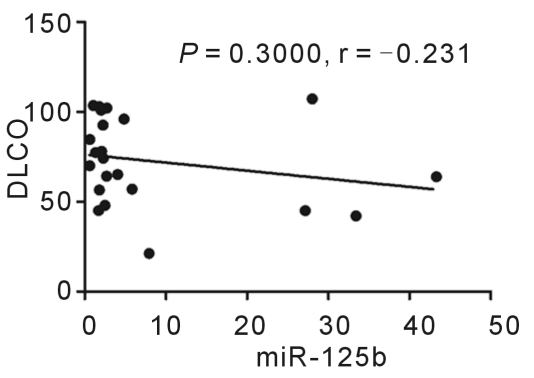

(d)

Figure 6. The correlations between miR-125b, miR-200c and pulmonary function. The levels of miRNAs were detected by QRT-PCR, and they were expressed as relative levels to U6. There were negative correlations between miR-125b, miR-200c, and FEV1 ( $P=$ $0.021,0.041, \mathrm{r}=-0.489,-0.438$, Spearman correlation) (Figure 6(a), Figure 6(b)). There was also negative correlation between miR-200c and DLCO $(P=0.023, \mathrm{r}=-0.483$, Spearman correlation), and there was no significant correlation between miR-125b and $\operatorname{DLCO}(P=0.300, \mathrm{r}=-0.231$, Spearman correlation) (Figure 6(c), Figure 6(d)).

associated with cell proliferation, differentiation and fibrosis [9] [15]-[21], were positively correlated with the severity of ILD in DM/PM, moreover, the two miRNAs were positively correlated with ILD-associated auto-antibody, anti-JO-1, and positively correlated with lung damage marker, SP-D. All these results suggest that miR-125b and miR-200c may play an important role in pathogenesis of ILD in DM/PM.

Lung fibrosis manifests an abnormal wound healing of AECs after damage. During the course, AECs undergo multiple changes and become mesenchymal cell features (termed EMT), and these mesenchymal cells produce excessive ECM and promote lung fibrosis [1] [22]. Many signal pathways were reported involved in the process of fibrosis, such as, TGF- $\beta$-Smad, MAPK, phosphoinositide 3-kinase (PI3K)-Akt, mTOR pathways [1]. Many miRNAs are involved in the process of lung fibrosis by regulating the expression of crucial genes in these pathways. MiR-125b plays crucial roles in different cellular processes, such as cell differentiation, proliferation and migration by targeting different genes, such as, ETS1, MMP11, VEGF, and so on [9]. MiR-200c represses EMT by targeting transcriptional repressors, including ZEB1 and ZEB2 [8] [23]. These miRNAs influence the process of lung fibrosis, including EMT, proliferation, differentiation of AECs to myofibroblasts, and migration of fibroblasts.

In current study, the levels of miR-125b and miR-200c were found higher in 
$\mathrm{DM} / \mathrm{PM}$, which indicates these miRNAs might participate in the development of $\mathrm{DM} / \mathrm{PM}$. In lung tissue, we found the levels of miR-125b and miR-200c were higher in bronchoalveolar cells than in PBMCs in DM/PM-ILD patients, meanwhile, there were positive correlation between miR-125b, miR-200c and SP-D (a marker of lung damage). So, the two miRNAs might be related to DM/PM-ILD.

Moreover, we found the levels of miR-125b and miR-200c were more higher in severe ILD group than non-ILD or mild ILD group and moderate ILD group, moreover, miR-200c was negatively correlated with the parameters of pulmonary function, FEV1, DLCO, and miR-125b was negatively correlated with FEV1, so, the two miRNAs were positively correlated with the severity of ILD, which confirmed the roles of miR-125b and miR-200c in ILD associated with $\mathrm{DM} / \mathrm{PM}$ further.

Some auto-antibodies were reported associated with ILD in IIM (idiopathic inflammatory myopathies), such as anti-JO-1, anti-SSA [24] [25] [26]. In current study, we also found that the patients with anti-JO-1 or anti-SSA all had ILD, however, the disease condition of ILD in patients with anti-JO-1 was more severe than patients with anti-SSA. Moreover, the levels of miR-125b and miR-200c were more higher than the whole median in patients with anti-JO-1 antibody, but it was not obvious in patients with anti-SSA antibody. Our results not only confirmed anti-JO-1 and anti-SSA are related to ILD in DM/PM, moreover, it seemed that there were positive correlation among miR-125b, miR-200c, anti-JO-1, and ILD in DM/PM, but the correlation was not obvious in patients with anti-SSA antibody.

TGF- $\beta$ is a pivotal upstream molecule of several signal pathways which lead to diverse fibrotic diseases, including lung fibrosis, such as TGF- $\beta$-Smad, PI3K-Akt, and mTOR pathways, etc [27] [28] [29] [30]. It was reported that miR-21 regulated TGF- $\beta$-Smad pathway [31], while it is not known whether miR-125 and (or) miR-200c work through the same pathway. In current study, we also found the concentration of TGF- $\beta 1$ in supernatant of BALF was significantly higher than in plasma, which supported the role of TGF- $\beta 1$ in ILD [31] [32]. However, No correlation was found between miR-125b, miR-200c and TGF- $\beta 1$ in BALF, and the reason may be these miRNAs don't work through the same pathway as miR-21.

Muscle enzyme is one of diagnostic criteria for DM/PM, however, it may be not positive related to ILD. In literature, it was reported that different types of IIM had different levels of severity for ILD, and CADM patients were more likely to have severe ILD [33] [34]. In current study, we found CPK was more higher in non or mild ILD group than mediate ILD group, however, there was no difference among other groups, and there was no difference for LDH among different level ILD groups either. So, the correlation between muscle enzymes and ILD seems complicated, which need an enlarged sample for future study.

It was not strange we didn't find the correlation between miR-125b, miR-200c, and CPK, LDH because of no correlation between ILD and muscle 
enzymes and positive correlation between ILD and miR-125b, miR-200c. Moreover, we didn't find the differences of miR-125b and miR-200c between CADM and non-CADM groups, and between CADM and typical DM groups either, the reason may be the small sample and the relatively homogeneous patients for our study, because most of the patients were CADM, the numbers of PM and typical DM patients were small.

Therapeutic schedule may respond the activity of disease to some extent in whole, however, we didn't find the differences of miR-125b and miR-200c between different therapeutic schedule groups, the reason may be the relative small sample and homogeneous patients (most patients had CADM), another reason may be no correlation between these miRNAs and disease activity.

There was two limitations for our study: the first is the relative small sample and homogeneous patients, which might explain no difference for the levels of miR-125b and miR-200c between different types of IIM groups, and between different therapeutic schedule groups; the second is we only tested one of antisynthetase antibodies (anti-JO-1antibody), and didn't test anti-MDA5 (melanoma differentiation associated gene 5) antibody because of capital shortage. Despite of those limitations, miR-125b and miR-200c were first reported related with ILD-associated autoantibody (anti-JO-1), the marker of lung damage (SP-D), and positively related with the severity of ILD in DM/PM.

\section{Conclusion}

In a word, our results support that $\mathrm{miR}-125 \mathrm{~b}$ and $\mathrm{miR}-200 \mathrm{c}$ are positively related with the damage and severity of ILD in DM/PM, and these miRNAs are important markers for clinical judgment of disease condition. This paper even provides evidence of target therapy for ILD in DM/PM in future. However, it is unknown about the detailed pathogenesis, which will be a direction for future study.

\section{Acknowledgements}

We thank all the nurses who helped to take blood specimens, and thank Zuo Ting from Anqing Municipal hospital, Anhui province, for her collecting clinical data, and Fang Xuan from Molecular Biology Lab of Anhui Provincial Hospital.

\section{Funding}

This work was supported by Science and Technology Hall of Anhui province, China, in 2012 (grant number 12070403052).

\section{References}

[1] Jimenez, S.A. and Piera-Velazquez, S. (2016) Endothelial to Mesenchymal Transition (EndoMT) in the Pathogenesis of Systemic Sclerosis-Associated Pulmonary Fibrosis and Pulmonary Arterial Hypertention. Myth or Reality? Matrix Biology, 51, 26-36. https://doi.org/10.1016/j.matbio.2016.01.012 
[2] Hostettler, K. (2016) Idiopathic Pulmonary Fibrosis-Pathogenesis and Therapeutic Concepts. Therapeutische Umschau, 73, 19-24. https://doi.org/10.1024/0040-5930/a000750

[3] Yoon, J.H., Abdelmohsen, K. and Gorospe, M. (2014) Functional Interactions among microRNAs and Long Noncoding RNAs. Seminars in Cell and Developmental Biology, 34, 9-14. https://doi.org/10.1016/j.semcdb.2014.05.015

[4] Jiang, X.P., Ai, W.B., Wan, L.Y., et al. (2017) The Roles of microRNA Families in Hepatic Fibrosis. Cell \& Bioscience, 7, 34. https://doi.org/10.1186/s13578-017-0161-7

[5] Wang, S., Wu, L., Du, L., et al. (2017) Reduction in miRNA-125b-5p Levels Is Associated with Obstructive Renal Injury. Biomedical Reports, 6, 449-454. https://doi.org/10.3892/br.2017.875

[6] Pottier, N., Cauffiez, C., Perrais, M., et al. (2014) FibromiRs: Translating Molecular Discoveries into New Anti-Fibrotic Drugs. Trends in Pharmacological Sciences, 35, 119-126. https://doi.org/10.1016/j.tips.2014.01.003

[7] Liang, H., Gu, Y., Li, T., et al. (2014) Integrated Analyses Identify the Involvement of microRNA-26a in Epithelial-Mesenchymal Transition during Idiopathic Pulmonary Fibrosis. Cell Death \& Disease, 5, e1238.

https://doi.org/10.1038/cddis.2014.207

[8] Yang, S., Banejee, S., de Freitas, A., et al. (2012) Participation of miR-200 in Pulmonary Fibrosis. The American Journal of Pathology, 180, 484-493.

https://doi.org/10.1016/j.ajpath.2011.10.005

[9] Sun, Y.M., Lin, K.Y. and Chen, Y.Q. (2013) Diverse Functions of miR-125b Family in Different Cell Contexts. Journal of Hematology \& Oncology, 6, 6. https://doi.org/10.1186/1756-8722-6-6

[10] Guo, Z., Gu, Y., Wang, C., et al. (2014) Enforced Expression of miR-125b Attenuates LPS-Induced Acute Lung Injury. Immunology Letters, 162, 18-26. https://doi.org/10.1016/j.imlet.2014.06.008

[11] Lim, J.U., Kang, H.S., Kim, Y.H., et al. (2017) Amyopathic Dermatomyositis Associated with Histopathological Findings of Organizing Pneumonia and Pulmonary Vasculitis. Balkan Medical Journal, 34, 374-377. https://doi.org/10.4274/balkanmedj.2016.1061

[12] Li, T., Guo, L., Chen, Z., et al. (2016) Pirfenidone in Patients with Rapidly Progressive Interstitial Lung Disease Associated with Clinically Amyopathic Dermatomyositis. Scientific Reports, 6, 33226. https://doi.org/10.1038/srep33226

[13] Travis, W.D., Costabel, U., Hansell, D.M., et al. (2013) An official American Thoracic Society /European Respiratory Society Statement: Update of the International Multidisciplinary Classification of the Idiopathic Interstitial Pneumonias. American Journal of Respiratory and Critical Care Medicine, 188, 733-748. https://doi.org/10.1164/rccm.201308-1483ST

[14] Jiang, Z., Tao, J.-H., Zuo, T., et al. (2016) The Correlation between miR-200c and the Severity of Interstitial Lung Disease Associated with Different Connective Tissue Diseases. Scandinavian Journal of Rheumatology, 46, 122-129.

[15] Liu, Z., Chen, X., Wu, Q., et al. (2016) miR-125b Inhibits Goblet Cell Differentiation in Allergic Airway Inflammation by Targeting SPDEF. European Journal of Pharmacology, 782, 14-20. https://doi.org/10.1016/j.ejphar.2016.04.044

[16] Wang, J., Luo, H., Xiao, Y., et al. (2016) miR-125b Inhibits Keratinocyte Proliferation and Promotes Keratinocyte Apoptosis in Oral Lichen Planus by Targeting 
MMP-2 Expression through PI3K/Akt/mTOR Pathway. Biomedicine \& Pharmacotherapy, 80, 373-380. https://doi.org/10.1016/j.biopha.2016.02.043

[17] Nagpal, V., Rai, R., Place, A.T., et al. (2016) MiR-125b Is Critical for Fibroblast-to-Myofibroblast Transition and Cardiac Fibrosis. Circulation, 133, 291-301. https://doi.org/10.1161/CIRCULATIONAHA.116.022627

[18] Jurmeister, S., Baumann, M., Balwierz, A., et al. (2011) MicroRNA-200c Represses Migration and Invasion of Breast Cancer Cells by Targeting Actin-Regulatory Proteins FHOD1 and PPM1F. Molecular and Cellular Biology, 32, 633-651. https://doi.org/10.1128/MCB.06212-11

[19] Cao, H., Jheon, A., Li, X., et al. (2013) The Pitx2:miR-200c/141:Noggin Pathway Regulates Bmp Signaling and Ameloblast Differentiation. Development, 140, 3348-3359. https://doi.org/10.1242/dev.089193

[20] Yu, J., Ohuchida, K., Mizumoto, K., et al. (2010) MicroRNA, hsa-miR-200c, Is an Independent Prognostic Factor in Pancreatic Cancer and Its Upregulation Inhibits Pancreatic Cancer Invasion but Increases Cell Proliferation. Molecular Cancer, 9, 1051-1058. https://doi.org/10.1186/1476-4598-9-169

[21] Rebustini, I.T., Hayashi, T., Reynold, A.D., et al. (2012) miR-200c Regulates FGFR-Dependent Epithelial Proliferation via Vldlr during Submandibular Gland Branching Morphogenesis. Development, 139, 191-202. https://doi.org/10.1242/dev.070151

[22] Willis, B.C., duBois, R.M. and Borok, Z. (2006) Epithelial Origin of Myofibroblasts during Fibrosis in the Lung. Proceedings of the American Thoracic Society, 3, 377-382. https://doi.org/10.1513/pats.200601-004TK

[23] Gregory, P.A., Bracken, C.P., Smith, E., et al. (2011) An Autocrine TGF-beta/ZEB/ miR-200 Signaling Network Regulates Establishment and Maintenance of Epithelial-Messenchymal Transition. Molecular Biology of the Cell, 22, 1686-1698. https://doi.org/10.1091/mbc.E11-02-0103

[24] Váncsa, A., Csípo, I., Németh, J., et al. (2009) Characteristics of Interstitial Lung Disease in SS-A Positive/Jo-1 Positive Inflammatory Myopathy Patients. Rheumatology International, 29, 989-994. https://doi.org/10.1007/s00296-009-0884-9

[25] Mimori, T., Nakashima, R. and Hosono, Y. (2012) Interstitial Lung Disease in Myositis: Clinical Subsets, Biomarkers, and Treatment. Current Rheumatology Reports, 14, 264-274. https://doi.org/10.1007/s11926-012-0246-6

[26] Ascheman, D.P. (2015) Role of Jo-1 in the Immunopathogenesis of the Anti-Synthetase Syndrome. Current Rheumatology Reports, 17, 56. https://doi.org/10.1007/s11926-015-0532-1

[27] Chen, X., Wang, H., Liao, H.J., et al. (2014) Integrin-Mediated Type II TGF- $\beta$ Receptor Tyrosine Dephosphorylation Controls SMAD-Dependent Profibrotic Signaling. Journal of Clinical Investigation, 124, 3295-3310. https://doi.org/10.1172/JCI71668

[28] Wang, P., Nie, X., Wang, Y., et al. (2013) Multiwall Carbon Nanotubes Mediate Macrophage Activation and Promote Pulmonary Fibrosis Through TGF- $\beta /$ Smad Signaling Pathway. Small, 9, 3799-3811. https://doi.org/10.1002/smll.201300607

[29] Li, H.Y., Zhang, Q.G., Chen, J.W., et al. (2013) The Fibrotic Role of Phosphatidylinositol-3-kinase/Akt Pathway in Injured Skeletal Muscle after Acute Contusion. International Journal of Sports Medicine, 34, 789-794. https://doi.org/10.1055/s-0032-1333284

[30] Kurita, Y., Araya, J., Minagawa, S., et al. (2017) Pirfenidone Inhibits Myofibroblast 
Differentiation and Lung Fibrosis Development during Insufficient Mitophagy. Respiratory Research, 18, 114. https://doi.org/10.1186/s12931-017-0600-3

[31] Liu, G., Friggeri, A., Yang, Y., et al. (2010) miR-21 Mediates Fibrogenic Activation of Pulmonary Fibroblasts and Lung Fibrosis. The Journal of Experimental Medicine, 207, 1589-1597. https://doi.org/10.1084/jem.20100035

[32] Grünert, S., Jechlinger, M. and Beug, H. (2003) Diverse Cellular Mechanisms Contribute to Epithelial Plasticity and Metastasis. Nature Reviews Molecular Cell Biology, 4, 657-665. https://doi.org/10.1038/nrm1175

[33] Nakashima, R., Imura, Y., Kobayashi, S., et al. (2009)The RIG-I-Like Receptor IFIH1/MDA5 Is a Dermatomyositis-Specific Autoantigen Identified by the Anti-CADM-140 Antibody. Rheumatology (Oxford), 49, 433-440. https://doi.org/10.1093/rheumatology/kep375

[34] Sato, S., Hirakata, M., Kuwana, M., et al. (2005) Autoantibodies to a 140-kd Polypeptide, CADM-140, in Japanese Patients with Clinically Amyopathic Dermatomyositis. Arthritis \& Rheumatology, 52, 1571-1576.

https://doi.org/10.1002/art.21023 


\section{Supplement}

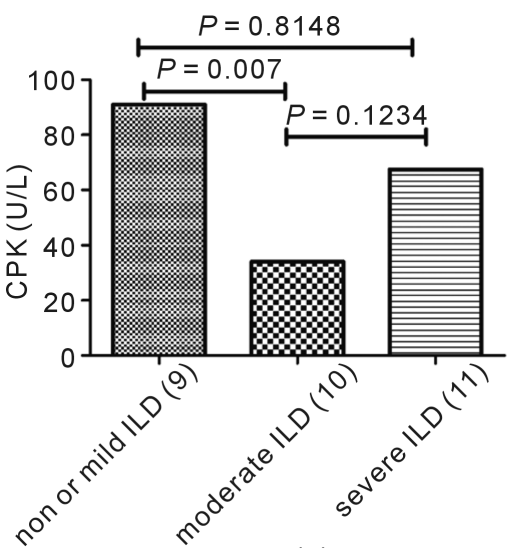

(a)

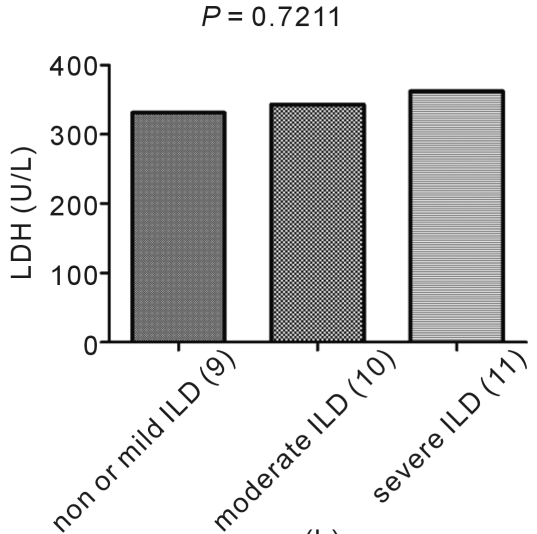

(b)

Figure S1. The correlation between muscle enzymes and the severity of ILD. The difference of CPK was significant among three groups of severity for ILD $(P=0.0321$, Kruskal-Wallis test), and the level in mild or non-ILD group was significantly higher than in moderate ILD group ( $P=0.007$, Mann Whitney test) (Figure S1(a)). The difference of LDH was not significant among three groups of severity for ILD $(P=0.7211$, Kruskal-Wallis test) (Figure S1(b)). 